\title{
Image Segmentation with Multilevel Threshold of Gray-Level \& Gradient-Magnitude Entropy Based on Genetic Algorithm
}

\author{
Z. Fu, J.F. He, R. Cui, Y. Xiang, S.L. Yi, S.J. Cao, Y.Y. Bao, K.K. Du, H. Zhang, J.X. Ren \\ Institute of Biomedical Engineering of college of Information Engineering and Automation Kunming University of Science and \\ Technology \\ KMUST Kunming, China
}

\begin{abstract}
Due to consider the gray level spatial distribution information, some image segmentation technologies based on entropy threshold can enhance the thresholding segmentation performance. However, they still cannot distinguish image edges and noise well. Even though GLGM(gray-level \& gradientmagnitude) entropy can effectively solve the problem, but it cannot segment effectively multi-objective and complex image. In this paper, a GLGM entropy fast segmentation method based on GA is presented by combining Real-code-GA and GLGM entropy, and the single threshold segmentation of GLGM entropy is further extended to multilevel threshold segmentation. Our method compared with GLGM entropy multi-threshold exhaustive method, the segmentation result obtained by our method is basically the same as the result obtained by exhaustive method.
\end{abstract}

Keywords-genetic algorithm; gray-level \& gradient-magnitude entropy; multi-threshold segmentation; image segmentation

\section{INTRODUCTION}

Image segmentation is a process technology dividing the image into several interest regional of specific and unique nature. One of the most widely used image segmentation methods is the threshold method [1].

In recent years, many threshold segmentation methods have been proposed, and the threshold method based on entropy has increasingly become the focus of attention. Pun [2] first of all used the entropy to implement the threshold segmentation; Kapur [3] put forward the improved algorithm for overcome the limitations of Pun theory; in order to solve the problem of Kapur theory did not consider the spatial distribution information of gray scale, Abutaleb [4] proposed a threshold segmentation method based on two-dimensional histogram entropy, which uses the average values of the corresponding pixel area to distinguish pixel gray value; in addition, Xiao [5] reported an entropy algorithm of spatial correlation histogram by observing the neighborhood pixels whether similar; Yimit [6] developed a novel entropy algorithm by introduced the information of gray-direction gradient. Xiao [7] presented an algorithm of GLGM entropy, which can accurately distinguish the edge and noise.However, for multi-threshold segmentation of the complex image, along with the increase of the threshold value, the GLGM entropy algorithm computational complexity will exponentially grows [8]. Genetic algorithm is a method by natural selection, hybridization and mutation to implement search best threshold.
It can provide powerful search capabilities even though under the situation of the search space is not continuous. The biggest advantage is that it can be adaptive search speed and avoid falling into local optimum [9].

In this paper, we proposed a new image segmentation method by combining with the genetic algorithm of real coding and the GLGM entropy algorithm, which can implement the multi-threshold segmentation of the complex image and multi-target images.

\section{GLGM ENTROPY MULTI-THRESHOLD SEGMENTATION BASED ON GENETIC ALGORITHMS}

\section{A. GLGM Entropy Segmentation Method and Extension}

GLGM entropy Xiao et al. proposed, the main idea that enhances the thresholding performance by embedding gray level occurrence and spatial distribution property based on the one-dimensional entropy. For an image $F=\{\mathrm{f}(\mathrm{x}, \mathrm{y}) \mid \forall \mathrm{x} \in\{1,2, \ldots \mathrm{M}\}, \forall \mathrm{y} \in\{1,2, \ldots \mathrm{N}\}\}$ of size $M \times N$,firstly, it will be processed by soble operator to gradient amplitude images namely $I\left(\mathrm{x}_{1}, \mathrm{y}_{1}\right)$, which the range of $\left(\mathrm{x}_{1}, \mathrm{y}_{1}\right)$ is $\left[g_{\min }, g_{\max }\right]$.Then, the gradient magnitude value range is partitioned into 88 segments uniformly with no overlap by a sequence of 9 Fibonacci numbers namely fibonacci $=[1,1,2,3,5,8,13,21,34]$.Lastly, the segments are merged from low value to high value area respectively to deposit in the "Fibonacci" quantization bins[7]. Meanwhile, the pixels can be mapped to different image hierarchies according to their gradient magnitude quantization values. GLGM histogram $p(\mathrm{~s}, \mathrm{q})$ is defined as:

$$
\begin{aligned}
& p(\mathrm{~s}, \mathrm{q})=\operatorname{Prob}(\mathrm{f}(\mathrm{x}, \mathrm{y})=\mathrm{s} \text { and } \mathrm{g}(\mathrm{x}, \mathrm{y})=\mathrm{q}) \\
& =\frac{\text { number of tuples }(\mathrm{f}(\mathrm{x}, \mathrm{y})=\mathrm{s}, \mathrm{g}(\mathrm{x}, \mathrm{y})=\mathrm{q})}{M \times N} \\
& s \in\{0,1,2, \ldots 255\}, \mathrm{q} \in\{1,2, \ldots 9\}
\end{aligned}
$$

Where $f(x, y)$ is the gray value, $g(x, y)$ represents the relevant Fibonacci quantization value of gradient magnitude.Single threshold GLGM entropy is defined as:

$$
\phi(t)=H(B)+H(F)
$$




$$
\begin{gathered}
H(\mathrm{~B})=-\sum_{s=0}^{t} \sum_{q=1}^{9} \frac{p(s, q)}{P(F)} \ln \left(\frac{p(s, q)}{P(F)}\right) \operatorname{Weight}(q) \\
H(F)=-\sum_{s=t+1}^{255} \sum_{q=1}^{9} \frac{p(s, q)}{P(B)} \ln \left(\frac{p(s, q)}{P(B)}\right) \operatorname{Weight}(q)
\end{gathered}
$$

Where $\phi(\mathrm{t})$ is GLGM entropy, ${ }^{H(\mathrm{~B})}$ is GLGM entropy of the image background, $H(\mathrm{~F})$ is GLGM entropy of image object, Weight(q) is the spatial property weighting function defined as Weight $(\mathrm{q})=\mathrm{q}^{3} . P(\mathrm{~B})$ and $P(\mathrm{~F})$ are respectively total probability of background histogram and objective histogram, defined as:

$$
\begin{aligned}
P(B) & =\sum_{s=0}^{t} \sum_{q=1}^{9} p(s, q) \\
P(F) & =\sum_{s=t+1}^{255} \sum_{q=1}^{9} p(s, q)
\end{aligned}
$$

The optimal threshold ${ }^{t}$ is chosen by maximizing $\phi(\mathrm{t})$.

Xiao's algorithm is used only for a single thresholding. When the complex and multi-objective image segmentation may result in loss of part of the signal or objective, Multithreshold segmentation can effectively solve this problem. So this paper presents the GLGM entropy single threshold extended to multi-thresholding. Correlation is defined as follows:

$$
\begin{gathered}
\phi\left(t_{1}, t_{2}, \ldots, t_{n}\right)=H(B)+H\left(F_{1}\right)+\ldots+H\left(F_{n}\right) \\
H(B)=-\sum_{s=0}^{t_{1}} \sum_{q=1}^{9} \frac{p(s, q)}{P(B)} \ln \left(\frac{p(s, q)}{P(B)}\right) \operatorname{Weight}(q) \\
H\left(F_{1}\right)=-\sum_{s=t_{1}+1}^{t_{2}} \sum_{q=1}^{9} \frac{p(s, q)}{P\left(F_{1}\right)} \ln \left(\frac{p(s, q)}{P\left(F_{1}\right)}\right) \operatorname{Weight}(q) \\
H\left(F_{n}\right)=-\sum_{s=t_{n}+1}^{255} \sum_{q=1}^{9} \frac{p(s, q)}{P\left(F_{n}\right)} \ln \left(\frac{p(s, q)}{P\left(F_{n}\right)}\right) \operatorname{Weight}(q)
\end{gathered}
$$

Where $\phi\left(t_{1}, t_{2}, \ldots, t_{n}\right)$ is GLGM entropy, $H(B)$ is GLGM entropy of the image background, $H\left(F_{1}\right) 、 H\left(F_{2}\right) \ldots H\left(F_{n}\right)$ is GLGM entropy of image object, $\operatorname{Weight}(q)$ is the spatial property weighting function defined as $\operatorname{Weight}(q)=q^{3} . P(B)$ is total probability of background histogram, $P\left(F_{1}\right)$ 、 $P\left(F_{2}\right) \ldots P\left(F_{n}\right)$ are respectively total probability of objective 1 to objective $\mathrm{n}$ histogram, defined as:

$$
\begin{gathered}
P(B)=\sum_{s=0}^{t_{1}} \sum_{q=1}^{9} p(s, q) \\
P\left(F_{1}\right)=\sum_{s=t_{1}+1}^{t_{2}} \sum_{q=1}^{9} p(s, q) \\
P\left(F_{n}\right)=\sum_{s=t_{n-1}+1}^{255} \sum_{q=1}^{9} p(s, q)
\end{gathered}
$$

$t_{1}, t_{2}, \ldots, t_{n}$ is Segmentation threshold, subject to $0<t_{1}<t_{2}<\ldots<t_{n}<L$. The optimal threshold $t_{1}, t_{2}, \ldots, t_{n}$ is chosen by maximizing $\phi\left(t_{1}, t_{2}, \ldots, t_{n}\right)$.

Single threshold extended to the multi-threshold segmentation can efficiently resolve complex and multi-target image segmentation problems.

\section{B. The Optimal Threshold Determined by Genetic Algorithm Automatically}

There are some problems such as population precocious, poor adaptive ability, slow convergence speed and the occasional converges to local optimal solution in traditional genetic algorithm. To solve these problems, we use tournament selection, simulated binary crossover and polynomial mutation [10] as genetic operator. This method is very effective in terms of constrained optimization, and can improve the convergence rate of genetic algorithms.

The process of simulation of binary crossover is randomly generated at the intersection of two parents ${ }^{x_{r, 1, k}}$ and ${ }^{x_{r, 2, k}}$, then generates two offspring $x_{1, k}$ and ${ }^{x_{2, k}}$ by formula (4).

$$
\begin{aligned}
& x_{1, k}=\frac{1}{2}\left[\left(1-\beta_{k i}\right) x_{r, 1, k}+\left(1+\beta_{k i}\right) X_{r, 2, k}\right] \\
& x_{2, k}=\frac{1}{2}\left[\left(1+\beta_{k i}\right) x_{r, 1, k}+\left(1-\beta_{k i}\right) x_{r, 2, k}\right]
\end{aligned}
$$

Calculate a spread factor as given in the following equation:

$$
\beta_{k i}=\left\{\begin{array}{c}
\left(2 \mu_{i}\right)^{\frac{1}{n_{c}+1}}, \mu_{i} \leq 0.5 \\
\left(\frac{1}{2\left(1-\mu_{i}\right)}\right)^{\frac{1}{n+1}}, \mu_{i}>0.5
\end{array}\right.
$$

$\mu_{i}$ is a random number of ${ }^{[0,1]} . \eta_{c}$ is the crossover index.

Polynomial mutation expression as follows:

$$
y_{i}^{(1, t+1)}=x_{i}^{(1, t+1)}+\left(x_{i}^{u}-x_{i}^{L}\right) \delta_{i}
$$

$y_{i}^{(1, t+1)}$ in the formula (6) represents the new offspring. ${ }^{x_{i}^{u}}$ and ${ }^{x_{i}^{L}}$ are the upper and lower limit values. The parameter $\delta_{i}$ is obtained from the polynomial probability distribution. The specific formula is as follows:

$$
P(\delta)=0.5\left(\eta_{m}+1\right)(1-|\delta|)^{\eta_{m}}
$$

The parameter $\delta_{i}$ is calculated

$$
\delta_{i}=\left\{\begin{array}{cc}
\left(2 \mathrm{r}_{i}\right)^{\frac{1}{\left(\eta_{m}+1\right)}}-1, & r_{i}<0.5 \\
1-\left[2\left(1-\mathrm{r}_{i}\right)\right]^{\frac{1}{\left(\eta_{m}+1\right)}}, & r_{i}>=0.5
\end{array}\right.
$$

Where $\eta_{m}$ is the mutation index. The algorithm steps are as follows: 
Step1: Initialization parameters: setting the number of populations pop , crossover probability $p_{c}$ and mutation probability $p_{m}$.

Step2: Randomly generated ${ }^{p o p}$ individual species, That is to say, get populations chromosome .Calculate the fitness function of chromosome, it gets $f_{1} \sim f_{\text {pop }}$.Determine the maximum number of iterations $g^{g e n_{\max }}$.Initialization $g e n=1$, start the iteration.

Step3 : Mating pool Initialization pool $=1$.Compare the size of any two fitness values, the bigger is copied to the next generation; $\quad$ pool $+1 \quad$,if $\quad$ pool $\leq \frac{p o p}{2} \quad$,repeat Step3.Get parent_chromosome whose individuals are $\frac{p o p}{2}$,go Step4。

Step4: Specify crossover index $\eta_{c}$, mutation index $\eta_{m}$, Initialization $N=1$; If $\operatorname{rand}(1)<p_{c}$,two individuals were randomly selected from the parent_chromosome, and get two offspring by Equation (4), calculate the corresponding value of the fitness function. If $\operatorname{rand}(1)>p_{c}$, one of individuals was randomly selected from the parent_chromosome , and get one offspring by Equation (6), calculate the corresponding value of the fitness function. $N+1$,if $N \leq \frac{p o p}{2}$,repeat Step4, else generating go Step5.

Step5 : Mix chromosome and offspring_chromosome, Get intermediate_chromosome .

Step6: Based on individual fitness function value selected pop outstanding individuals in intermediate_chromosome, get chromosome.

Step7: $\quad$ gen +1 ,if $g e n \leq$ gen $n_{\max }$ go Step4, else go Step8.

Step8 : Determine the optimal threshold and segment mage.

\section{EXPERIMENTAL RESULTS AND ANALYSIS}

A. Multi-Threshold Segmentation Experiments of This Method

In this paper, two images were used for genetic algorithms GLGM entropy threshold three, four and five threshold segmentation. The optimal parameter combination of this algorithm is tested through a series of different parameter settings. Finally, arguments are set as follows: The number of population $\quad$ op $=600$,iterations gen $=50$,cross Index $\eta_{c}=15$,mutation Index $\eta_{m}=20$, crossover probability $p_{c}=0.9$ and mutation probability $p_{m}=0.1$.Two images segmentation results are shown in Figure I, From top to bottom, respectively are elaine figure and goldhill figure; From left to right, original, 3,4,5 threshold segmentation results respectively. We can see that along with the increase of the number of threshold can be separated for more details in the image. In addition, Table I shows the average threshold obtained in the test 50 times and compared with a threshold value exhaustive method. The threshold result obtained by our method is basically the same as the result obtained by exhaustive method.

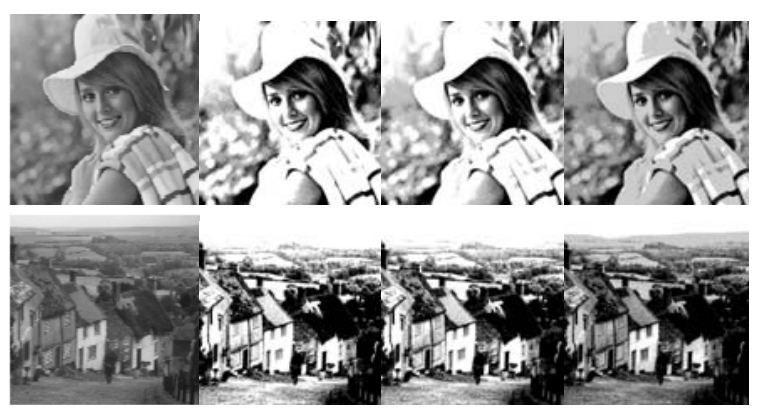

FIGURE I . SEGMENTATION RESULTS OF OUR METHOD.

TABLE I . AVERAGE THRESHOLDS OF DIFFERENT SEGMENTATION ALGORITHM.

\begin{tabular}{llll}
\hline Image & $\begin{array}{l}\text { Number } \\
\text { of } \\
\text { thresholds }\end{array}$ & Our method & $\begin{array}{l}\text { Exhaustive } \\
\text { method }\end{array}$ \\
\hline elaine & 3 & $(80,104,130)$ & $(80,104,130)$ \\
$250 * 250$ & 4 & $(75,95,116,143)$ & $(75,95,115,142)$ \\
& 5 & $(76,97,119,149,216)$ & $(77,98,119,149,216)$ \\
goldhill & 3 & $(82,98,118)$ & $(82,98,118)$ \\
$250 * 250$ & 4 & $(79,93,109,128)$ & $(78,93,108,127)$ \\
& 5 & $(82,98,116,138,192)$ & $(82,98,117,138,192)$ \\
\hline
\end{tabular}

B. Peak SNR and Uniformity Measurements Compare

In order to evaluate the proposed algorithm, Peak SNR[8] and uniformity measurements[10] are used to quantitatively analyze the effect of image segmentation. The value of the uniformity measure $\mathrm{u}$, is lie in between 0 and 1 . If $\mathrm{u}$ is close to 1 , the threshold segmentation of image means the better results. Peak SNR and uniformity measurements obtained experimental results as shown in Table II . 
TABLE II. PEAK SIGNAL-TO-NOISE RATIO AND UNIFORMITY MEASUREMENT OF THREE SEGMENTATION METHODS.

\begin{tabular}{cccccc}
\hline Image & $\begin{array}{c}\text { Number } \\
\text { of } \\
\text { thresholds }\end{array}$ & \multicolumn{2}{c}{ Our method } & \multicolumn{2}{c}{ Exhaustive method } \\
& & PSNR/dB & $\begin{array}{c}\text { Uniformity } \\
\text { Measure }\end{array}$ & PSNR/dB & $\begin{array}{c}\text { Uniformity } \\
\text { Measure }\end{array}$ \\
\hline elaine & 3 & 24.9544 & 0.95098 & 24.9544 & 0.95098 \\
$512 * 512$ & 4 & 27.192 & 0.95541 & 27.192 & 0.95541 \\
& 5 & 40.5414 & 0.97676 & 40.5414 & 0.97676 \\
goldhill & 3 & 25.2653 & 0.92674 & 25.2653 & 0.92674 \\
$512 * 512$ & 4 & 26.446 & 0.92885 & 26.446 & 0.92885 \\
& 5 & 35.4462 & 0.97429 & 35.4462 & 0.97429 \\
\hline
\end{tabular}

The results in table II shows that with the increase of the threshold, the image PSNR and uniformity measurements value is higher. The value obtained by our method is basically the same the result obtained by exhaustive method by Table2.

\section{CONCLUSIONS}

Due to consider the gray level spatial distribution information, some image segmentation technologies based on entropy threshold can enhance the thresholding segmentation performance. However, they still cannot distinguish image edges and noise well. Even though GLGM entropy can effectively solve the problem, but it cannot segment effectively multi-objective and complex image. In this paper, A GLGM entropy fast segmentation method based on GA is presented by combining Real-codeGA and GLGM entropy, and the single threshold segmentation of GLGM entropy is further extended to multilevel threshold segmentation. Our method compares GLGM histogram entropy exhaustive method with peak signal-to-noise ratio and uniformity measurement. The results show that the segmentation result obtained by our method is basically the same as the result obtained by exhaustive method.

\section{ACKNOWLEDGEMENTS}

This study was supported by the National Natural Science Foundation of China (No.11265007), and Supported by Scientific Research Foundation for Returned Scholars, Ministry of Education of china (No. 2010-1561).

\section{REFERENCES}

[1] Pedram G, Micael S C, Benediktsson J A et al.. An efficient method for segmentation of images based on fractional calculus and natural selection[J].Expert Systems with Applications, 2012,(39),pp. 1240712417.

[2] Pun T, et al.. Entropy thresholding: A new approach[J]. Computer Vision Graphics and Image Processing, 1981(16),pp. 210-239.

[3] Kapur J.N., Sahoo, P. K. et al.. A new method for gray-level picture thresholding using the entropy of the histogram[J]. Computer Vision Graphics Image Processing, 1985, 2:273-285.

[4] Abutaleb A S, et al.. Automatic thresholding of gray-level pictures using two-dimensional entropy[J]. Compute. Vis .Graphics Image Process.1989, 47(1),pp.22-32.

[5] Y Xiao,Z Cao,S Zhong, New entropic thresholding approach using gray-level spatial correlation histogram[J], Opt. Eng.2010,49(12),pp.127007.

[6] Yimit A, Hagihara Y, Miyoshi T, et al..2-D direction histogram based entropic thresholding[J]. Neurocomputing, 2013,120(23),pp.287-297.

[7] Y Xiao,Z Cao,S Zhong, Entropic image thresholding based on GLGM histogram[J]. Pattern Recognition Letters, 2014(40),pp.47-50.

[8] K CHENG,F CHENG, DAI M et al.. fast image segmentation with multilevel threshold of two-dimensional entropy based on firefly algorithm [J]. optics and Precision Engineering, 2014,22(2),pp. 05170513. (in Chinese)

[9] H ZH ZHANG,CH B XIANG,SONG J ZH, et al.. Application of improved adaptive genetic algorithm to image segmentation in realtime [J]. optics and Precision Engineering,2008.16(2),pp.333-337.(in Chinese)

[10] Manikandan S, Ramar K, Multilevel thresholding for segmentation of medical brain images using real coded genetic algorithm[J].Measurement, 2014(47),pp.558-568. 